\title{
Correlation of Preoperative Serum Tumor Markers with Clinicopathological Features and Prognosis in Breast Cancer
}

\author{
Yu-hong Zheng ${ }^{1}$, Yan Chen ${ }^{1}$, Min-hua Hu${ }^{1}$, Ying-ying $\operatorname{Lin}^{1} \&$ Yan-song Chen ${ }^{1}$ \\ 1 Clinical Laboratory Department, Laboratory of Biochemistry and Molecular Biology Research, Fujian \\ Provincial Cancer Hospital of Fujian Medical University Teaching Hospital, Fujian, China \\ Correspondence: Yan Chen, Clinical Laboratory Department, Laboratory of Biochemistry and Molecular \\ Biology Research, Fujian Provincial Cancer Hospital of Fujian Medical University Teaching Hospital, 420 Fuma \\ Road, Fuzhou, Fujian 350014, China. Tel: 86-591-8366-0063-8270. E-mail: yanc99@sina.com
}

Received: March 25, 2012 Accepted: April 5, 2012 Online Published: May 1, 2012

doi:10.5539/cco.vln1p124 URL: http://dx.doi.org/10.5539/cco.v1n1p124

\begin{abstract}
Background: Serologic tumor markers such as CA15-3, CEA and TPS have been used for decades to manage patients with breast cancer. Despite the frequent use of these markers, the prognostic significance of these markers remains indeterminate. Patients and methods: We retrospectively analyzed and followed up a set of 361 patients with Stages I III breast cancer, from January 2001 to February 2011. Serumal CA15-3 and CEA levels were measured by electrochemistry (ELC), and serumal TPS was analyzed by enzyme-linked immunosorbent assays (ELISA). We explored the relationship between preoperative serologic tumor markers and clinicopathological parameters, the correlation between CA15-3, CEA or TPS levels with overall survival, and their impact on prognosis. Results: First, at the univariate analysis, higher preoperative serum CA15-3 was significantly associated with older age of onset ( $\geq 45$ years, $\mathrm{P}=0.049$ ), and the expression of TPS was related with some traditional prognostic factors such as tumor size $(\mathrm{P}=0.030)$, histologic grade $(\mathrm{P}=0.001)$ and lymph node status $(\mathrm{P}=0.008)$. Second, overall survival were significantly shorter among patients with elevated preoperative serum CA15-3 ( $\mathrm{P}=0.000)$ or TPS $(\mathrm{P}=0.038)$ respectively. Finally, multivariate Cox regression indicated that histological grading $(\mathrm{P}=0.028)$, estrogen receptor $(\mathrm{ER})$ expression $(\mathrm{P}=0.001)$ and elevated preoperative values of CA 15-3 $(\mathrm{P}=0.015)$ were independent prognostic factors for overall survival. Conclusions: Our study demonstrates that higher expression of preoperative serum CA15-3 or TPS was closely correlated with clinicopathology and overall survival, and CA15-3 before treatment can be used as an independent prognostic parameter in patients with primarily breast cancer. However, serum CEA had poor correlation with clinical prognostic factors.
\end{abstract}

Keywords: breast cancer, tumor markers, clinicopathological, prognosis

\section{Introduction}

Breast cancer is a significant health problem world wide, mainly affecting women, the incidence of breast cancer has increased rapidly over the past few decades. The American Cancer Society estimated that 226,870 new female cases of invasive breast cancer will be diagnosed and 39,510 will die of breast cancer in the United States in 2012 (Siegel, Naishadham, \& Jemal, 2012). So breast cancer has become the most common malignancy in women, and was second only to lung cancer as a cause of cancer death. However, in contrast to other malignancies, breast cancer is potentially curable because of the existence of effective treatment modalities and favorable clinical or pathobiological tumor features.

Conceptually, the need for and selection of various local or systemic therapies are based on a number of prognostic and predictive factors. These factors contribute to the determination of the stage of disease, assist in the estimation of the risk that the cancer will recur, and provide information that predicts response to therapy. At present, for breast cancer, these factors include tumor histology, clinical and pathologic characteristics of the primary tumor, axillary node status, hormone receptor content, presence or absence of detectable metastatic disease, patient co-morbid conditions, patient age, and menopausal status. Besides that, several biochemical factors such as the serum tumor markers have been widely assessed as prognostic factors in breast cancer. However, the role of serum markers is less well established owing to the unsatisfactory evidence.

Although a large number of serum tumour markers have been proposed for breast cancer, but among them, only 
CA15-3 and CEA or cytokeratins (i.e. TPA, TPS or Cyfra 21-1) are the most commonly used in clinical practice. Carbohydrate antigen (CA) 15-3 is well-characterized assay in peripheral blood that allow the detection of circulating MUC-1 antigen. And MUC-1, which is one of cell surface associated mucin, is aberrantly over expressed in many adenocarcinomas especially breast cancer. Therefore, higher level of CA15-3 may be associated with larger burden of occult disease and poor outcome (Park et al., 2008). Carcinoembryonic antigen (CEA) is the most widely used marker in monitoring the clinical course of patients with cancer. Several authors have shown that an increase or a decrease in the CEA level may reflect the status of disease progression or regression (Uehara et al., 2008; Yerushalmi et al., 2011). Moreover, cytokeratin is an acid type intermediate filament protein detected in various types of human cancer. Tissue polypeptide specific antigens (TPS), which measures $\mathrm{M}_{3}$ antigenic determinant associated with human cytokeratin 18, is a marker of tumor cell activity in contrast to markers related to tumor burden (Rydlander et al., 1996). In breast cancer management, there is an improved effectiveness can be seen when TPS is used in combination with CA15-3 (Hwa et al., 2008).

Whether measuring these proteins in the serum at the time of primary intervention is a reliable method for monitoring prognosis? In the present study, we retrospectively analyzed the relationship between traditional prognostic factors and the three serum markers; evaluated the correlation between the markers and overall survival; and related preoperative levels of those markers to patient outcome using multivariate analysis.

\section{Patients and Methods}

\subsection{Selection of Patients}

All primary breast cancer patients, who treated at Fujian Provincial Cancer Hospital from January 2001 to December 2004, were considered for inclusion in this retrospective study. That means patients were excluded if any other malignancy was known from their history or if investigations at the time of diagnosis revealed evidence of metastases from other tissues, and or if them had been interfered. Finally, a total of 361 patients fulfilled these criteria, the average age of them was $47.1 \pm 9.2$ years (range 21 77 years), and the follow up period ranged from January 2001 to February 2011. Most parts of patients had their serum CA15-3 ( $\mathrm{n}=360$ ), CEA $(n=359)$ and TPS $(n=305)$ concentrations measured prior to therapy. Patient basic information such as name, age and menopausal status were evaluated at the time of diagnosis. General clinico-pathological parameters such as tumor size, lymph node status, histological grading (in the Nottingham Grading System), hormone receptor status (using $\geq 1 \%$ positive nuclear staining as cut off value) and CerbB- 2 expression (membrane staining $\geq 10 \%$ was used as positive critical value) were estimated after surgery. The final data set is summarized in Table 1. 
Table 1. Patient and disease characteristics of the primary breast cancer patients $(n=361)$

\begin{tabular}{|c|c|c|}
\hline Characteristics (N) & $\mathbf{N}$ & $\%$ \\
\hline \multicolumn{3}{|l|}{ Menopausal status (361) } \\
\hline Premenopause & 240 & 66.5 \\
\hline Postmenopause & 121 & 33.5 \\
\hline \multicolumn{3}{|l|}{ Age (361) } \\
\hline$\geq 45$ years & 216 & 59.8 \\
\hline$<45$ years & 145 & 40.2 \\
\hline \multicolumn{3}{|l|}{ Tumor size (361) } \\
\hline$<2 \mathrm{~cm}$ & 77 & 21.3 \\
\hline $2 \sim 5 \mathrm{~cm}$ & 206 & 57.1 \\
\hline$>5 \mathrm{~cm}$ & 78 & 21.6 \\
\hline \multicolumn{3}{|l|}{ Lymph node involvement (360) } \\
\hline 0 & 128 & 35.5 \\
\hline $1 \sim 3$ & 105 & 29.2 \\
\hline$>3$ & 127 & 35.3 \\
\hline \multicolumn{3}{|l|}{ Histologic grade (361) } \\
\hline I & 45 & 12.5 \\
\hline II & 225 & 62.3 \\
\hline III & 91 & 25.2 \\
\hline \multicolumn{3}{|l|}{ ER expression (361) } \\
\hline Negative & 179 & 49.6 \\
\hline Positive & 182 & 50.4 \\
\hline \multicolumn{3}{|l|}{ PR expression (361) } \\
\hline Negative & 166 & 46.0 \\
\hline Positive & 195 & 54.0 \\
\hline \multicolumn{3}{|l|}{ CerbB-2 expression (181) } \\
\hline Negative & 27 & 14.9 \\
\hline Positive & 154 & 85.1 \\
\hline \multicolumn{3}{|l|}{ CA15-3 (360) } \\
\hline$<30 \mathrm{U} / \mathrm{ml}$ & 324 & 90.0 \\
\hline$\geq 30 \mathrm{U} / \mathrm{ml}$ & 36 & 10.0 \\
\hline \multicolumn{3}{|l|}{ CEA (359) } \\
\hline$<2.5 \mu \mathrm{g} / \mathrm{L}$ & 331 & 92.2 \\
\hline$\geq 2.5 \mu \mathrm{g} / \mathrm{L}$ & 28 & 7.8 \\
\hline \multicolumn{3}{|l|}{ TPS (305) } \\
\hline$<80 \mathrm{AU} / \mathrm{ml}$ & 110 & 36.1 \\
\hline$\geq 80 \mathrm{AU} / \mathrm{ml}$ & 195 & 63.9 \\
\hline
\end{tabular}

ER: estrogen receptor; PR: progesterone receptor; CA15-3: cancer antigen 15-3; CEA: carcinoembryonic antigen; TPS: tissue polypeptide specific antigens.

The first line treatment with curative intent of primary breast cancer patients was either modified radical mastectomy or lumpectomy and axillary lymph node dissection. Soon after primary surgery, radiotherapy and appropriate adjuvant chemotherapy or hormone therapy was administered as indicated based on the international guidelines (NCCN - Breast Cancer ${ }^{2001-2004}$ ). Clinical follow-up included history taking, physical examination, and laboratory tests (including liver function test, complete blood count, CA15-3, CEA and TPS etc.), chest radiography, breast molybdenum target and abdominal ultrasonography every $4 \sim 6$ months, and bone scan every $6 \sim 12$ months, for detection of local or distant relapse. Additional computed tomography and radiography were obtained as necessary. Pay a return visit by telephone and letter, a number of cases data regarding relapse or 
death were available.

\subsection{Measurement of CEA, CA15-3 and TPS Expression}

Serumal CA15-3 and CEA concentrations were determined by automated test systems (ROCHE Analytics Modular E170, Germany) using electrochemistry (ELC) and the cut-off point for CA15-3 and CEA was 30 U/ml and $2.5 \mu \mathrm{g} / \mathrm{L}$, respectively. Serumal TPS was analyzed by commercial enzyme-linked immunosorbent assay (ELISA) kits (BEKI Diagnostics AB, Sweden), and the normal range was 0 to $80 \mathrm{AU} / \mathrm{ml}$.

\subsection{Statistical Analysis}

Data are expressed as means or numbers (\%). A Mean \pm Standard Deviation program calculated the mean values of the CA15-3, CEA and TPS levels. In the description of correlation between CA15-3, CEA and TPS levels with other clinicopathological variables, categorical variables were compared by $\chi^{2}$ analysis (chi-square test) or Fisher's exact test, and continuous variables were compared by Wilcoxon rank-sum test. The univariate survival curve of overall survival (OS), which was estimated using the Kaplan-Meier type, determined whether the risk of OS varied depending on the tumor marker's status, and were compared using log-rank test. Multivariate Cox regression analysis was performed to identify those parameters having an independent significant influence on OS and to calculate the hazard ratios. All statistical analysis was performed with the SPSS software package (version 13.0 for Windows), and a P value of less than 0.05 was considered statistically significant.

\section{Results}

Until the end of our study, there were 70 patients died from the disease, all cases were followed up except 11 . The average follow-up time was $58.8 \pm 32.1$ months (range 1 121 months). Patient demographics are listed in Table 1. The mean \pm SD. of preoperative serum CA15-3, CEA and TPS concentrations in breast cancer patients, who were took in this study, were $20.86 \pm 34.62 \mathrm{U} / \mathrm{ml}, 1.56 \pm 8.18 \mu \mathrm{g} / \mathrm{L}$, and $203.20 \pm 352.07 \mathrm{AU} / \mathrm{ml}$, respectively. Elevated CA15-3, CEA and TPS levels were identified in 36 (10.0\%), 28 (7.8\%) and 195 (63.9\%) patients, respectively.

\subsection{Association between the Three Serum Markers and Clinicopathological Variables}

Table 2 summarizes the associations between these tumor markers and other clinicopathological traditional prognostic factors such as tumour size, lymph node status, histological grading and hormone receptor status. As shown in Table 2, it is clear higher CA15-3 expression was significantly associated with older age ( $\mathrm{P}=0.049)$; increased TPS was significantly correlated with larger tumor size, higher histologic grade and greater lymph node metastases $(\mathrm{P}=0.030, \mathrm{P}=0.001$ and $\mathrm{P}=0.008)$; and $\mathrm{CEA}$ was not related with any clinical pathological parameters. In addition, it is interestingly that, none of the serum markers were correlated with menopausal status and immunohistological subtypes (hormone receptors positive or CerbB-2 positive). 
Table 2. Correlation between serum CA15-3, CEA, TPS and other clinicopathological variables

\begin{tabular}{|c|c|c|c|c|c|c|}
\hline \multirow[b]{2}{*}{ Parameter } & \multicolumn{2}{|c|}{ CA153 } & \multicolumn{2}{|l|}{ CEA } & \multicolumn{2}{|l|}{ TPS } \\
\hline & $\begin{array}{c}\text { Positive/total } \\
\text { (\%) }\end{array}$ & $\boldsymbol{P}$ & $\begin{array}{c}\text { Positive/total } \\
\text { (\%) }\end{array}$ & $\boldsymbol{P}$ & $\begin{array}{c}\text { Positive/total } \\
\text { (\%) }\end{array}$ & $\boldsymbol{P}$ \\
\hline Patients enrolled & 360 & & 359 & & 305 & \\
\hline \multicolumn{7}{|l|}{ Menopausal status } \\
\hline Premenopause & $19 / 239(7.9)$ & 0.068 & $22 / 238(9.2)$ & 0.152 & $128 / 204(62.7)$ & 0.432 \\
\hline Postmenopause & $17 / 121(14.0)$ & & $6 / 121(5.0)$ & & $68 / 101(67.3)$ & \\
\hline \multicolumn{7}{|l|}{ Age (years) } \\
\hline$<45$ & $9 / 145(6.2)$ & 0.049 & $8 / 144(5.6)$ & 0.194 & $75 / 124(60.5)$ & 0.254 \\
\hline$\geq 45$ & $27 / 215(12.6)$ & & 20/215(9.3) & & $121 / 181(66.9)$ & \\
\hline \multicolumn{7}{|l|}{ Tumor size (cm) } \\
\hline$<2$ & $5 / 77(6.5)$ & 0.416 & $5 / 76(6.6)$ & 0.174 & $36 / 66(54.5)$ & 0.030 \\
\hline $2 \sim 5$ & $21 / 205(10.2)$ & & $13 / 205(6.3)$ & & $111 / 175(63.4)$ & \\
\hline$>5$ & $10 / 78(12.8)$ & & $10 / 78(12.8)$ & & 49/64(76.6) & \\
\hline \multicolumn{7}{|c|}{ Lymph node $^{+}$status } \\
\hline 0 & $10 / 127(7.9)$ & 0.521 & $7 / 126(5.6)$ & 0.494 & $53 / 104(51.0)$ & 0.001 \\
\hline $1 \sim 3$ & $13 / 105(12.4)$ & & 9/105(8.6) & & $60 / 89(67.4)$ & \\
\hline$>3$ & $13 / 128(10.2)$ & & $12 / 128(9.4)$ & & $83 / 112(74.1)$ & \\
\hline \multicolumn{7}{|l|}{ Histologic grade } \\
\hline I & $3 / 45(6.7)$ & 0.605 & $2 / 44(4.5)$ & 0.187 & $16 / 38(42.1)$ & 0.008 \\
\hline II & $22 / 224(9.8)$ & & $15 / 224(6.7)$ & & $126 / 190(66.3)$ & \\
\hline III & 11/91(12.1) & & 11/91(12.1) & & $54 / 77(70.1)$ & \\
\hline \multicolumn{7}{|l|}{ ER receptor } \\
\hline Negative & 17/178(9.6) & 0.779 & $14 / 178(7.9)$ & 0.963 & $87 / 145(60.0)$ & 0.139 \\
\hline Positive & 19/182(10.4) & & 14/181(7.7) & & $109 / 160(68.1)$ & \\
\hline \multicolumn{7}{|l|}{ PR receptor } \\
\hline Negative & $16 / 165(9.7)$ & 0.860 & $12 / 165(7.3)$ & 0.731 & $83 / 137(61.5)$ & 0.226 \\
\hline Positive & 20/195(10.3) & & $16 / 194(8.2)$ & & $113 / 168(67.3)$ & \\
\hline \multicolumn{7}{|c|}{$\begin{array}{l}\text { CerbB-2 expression } \\
\text { (181) }\end{array}$} \\
\hline Negative & $3 / 27(11.1)$ & 0.392 & $2 / 26(7.7)$ & 1.000 & $16 / 26(61.5)$ & 0.983 \\
\hline Positive & 9/154(5.8) & & $12 / 154(7.8)$ & & $84 / 136(61.8)$ & \\
\hline
\end{tabular}

ER: estrogen receptor; PR: progesterone receptor; CA15-3: cancer antigen 15-3; CEA: carcinoembryonic antigen; TPS: tissue polypeptide specific antigens.

\subsection{Association between the Three Serum Markers and Overall Survival}

Kaplan-Meier curves for overall survival (OS) are shown in Figures 1-3, where the solid line represents patients with normal level and the dash line represents patients with elevated levels. A statistic $\mathrm{P}$ value indicated the 
statistical significance of differences in CA15-3 or TPS groups. Patients with normal levels of CA15-3 or TPS had a particularly favorable outcome compared with patients with elevated levels. That is to say increasing CA15-3 or TPS levels represent a higher rate of disease recurrence and mortality, and thus a poorer prognosis $(\mathrm{P}=0.000, \mathrm{P}=0.038)$. However, preoperative $\mathrm{CEA}$ had poor correlation with overall survival.

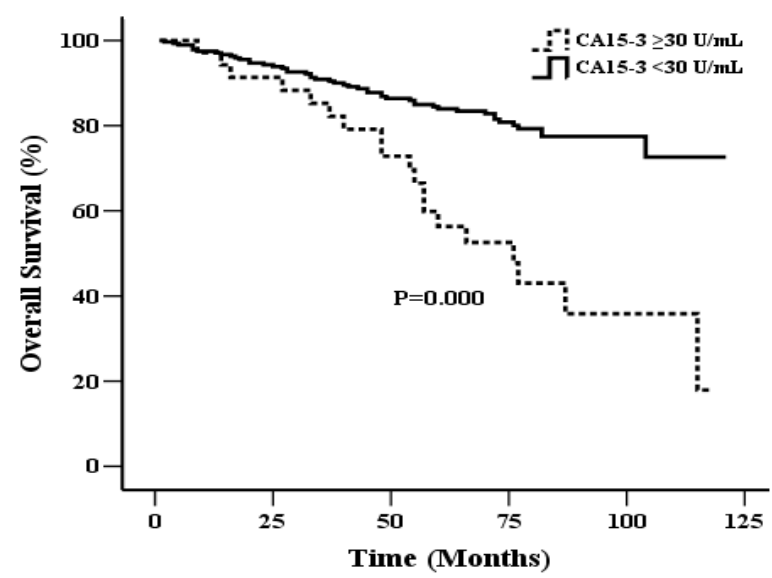

Figure 1. Overall survival curves between the normal and the elevated groups according to preoperative CA15-3 levels

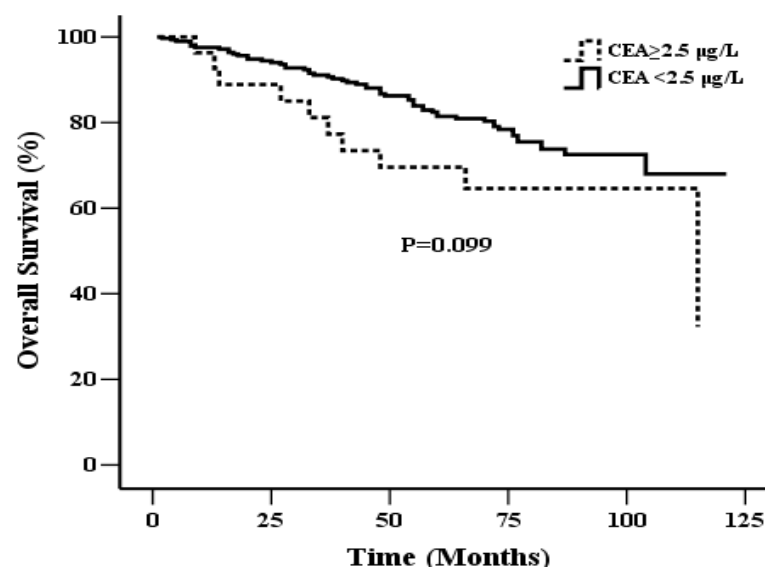

Figure 2. Overall survival curves between the normal and the elevated groups according to preoperative CEA levels

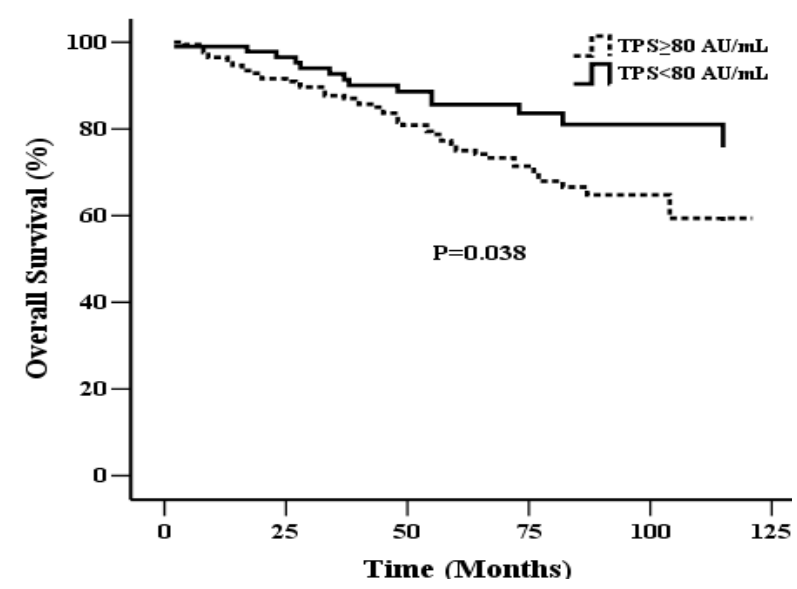

Figure 3. Overall survival curves between the normal and the elevated groups according to preoperative TPS levels 


\subsection{Association between the Three Serum Markers and Patient Outcome}

Hazard ratios analysis was performed using multivariate Cox regression, and the following variables were included in the model: menopausal status, age, tumor size, lymph node involvement, histologic grade, hormone receptor status, Cerb-B expressing and the three serumal markers preoperative expression. As shown in Table 3, histologic grade, estrogen receptor (ER) expression and elevated preoperative values of CA15-3 were independent prognostic factors for overall survival (OS) $(\mathrm{P}=0.028, \mathrm{P}=0.001$ and $\mathrm{P}=0.015)$, while histologic grade and increased preoperative CA15-3 were risk factor and positive estrogen receptor was safety factor. In addition, other factors were not associated with patient outcome.

Table 3. Cox proportional hazards regression analysis for overall survival (OS)

\begin{tabular}{lcccc}
\hline \multicolumn{1}{c}{ Factor } & B & $\operatorname{Exp(B)}$ & $\mathbf{9 5 \% C I ~ f o r ~} \operatorname{Exp(B)}$ & P \\
\hline Histologic grade* & 0.790 & 2.203 & $1.091 \sim 4.448$ & $\mathbf{0 . 0 2 8}$ \\
ER expression* & -1.311 & 0.270 & $0.125 \sim 0.579$ & $\mathbf{0 . 0 0 1}$ \\
Preoperative CA15-3* & 1.245 & 3.472 & $1.276 \sim 9.445$ & $\mathbf{0 . 0 1 5}$ \\
\hline
\end{tabular}

* Significantly different from reference group $(\mathrm{P}<0.05)$.

ER: estrogen receptor; PR: progesterone receptor; CA15-3: cancer antigen 15-3; CEA: carcinoembryonic antigen; TPS: tissue polypeptide specific antigens.

\section{Discussion}

Currently, there are multimodal therapies available for advanced breast cancer, but the degree and duration of response vary widely from patient to patient. Thus, the major challenge for physicians is to find a method helps in the establishment of individualized health care programmes. Which may decide whom should have a shorter interval of mammography or ultrasonography examination, whom should receive an invasive procedure for tissue proof, and whom should be advised to undergo lymph node dissection more extensively, rather than sentential lymph node sampling to prevent a false negative result. Risk profiles for individual patients at the initial diagnosis (early prediction) to be considered as informative and usable for further determination under therapy, which has already been paid more and more attention.

Despite continuous interest in early prediction, which has relevance to daily practice, very little is known about this topic. In the current world, histological and radiological examinations are routinely measured by the majority of physicians in prognostic evaluation of breast cancer patients. Although the histopatholgical examination is gold standard in any malignancy, it is sometimes difficult to perform examination efficiently in asymptomatic patients with microlesions. In addition, the peak incidence occurs in women aged 40-50 years when the breast tissue is still dense and the sensitivity of mammography is relatively low, and the false negative rate of mammography is above $20 \%$ (Ahn et al., 2006). In this situation, good serum markers for asymptomatic breast cancer are useful for identifying minute cancers and false negative cases, and for reducing the discomfort and cost of invasive procedures for false positive cases (Elmore et al., 1998). Therefore, quantitative detections of serum markers are often used in breast cancer as noninvasive tools to assess tumor burden.

However, the use of serum tumor markers faces several issues and unanswered questions: their specificity and sensitivity are considered as low, and no clear consensus exists on what threshold and /or variation should be considered clinically significant, and the biological heterogeneity that could not be detected by routine tests, and so on. For these reasons, there are some controversies internationally about the application of tumor markers in estimation of breast cancer patients. The European Group on Tumor Markers (EGTM, 2005) Panel considers that preoperatively elevated levels of either CA 15.3 or CEA are associated with adverse outcome in patients with breast cancer, and their use in combination with established prognostic factors is recommended. The Clinical Guidelines of American Society of Clinical Oncology (ASCO, 2007) also indicated that for monitoring patients, CA15-3 or CEA can be used in conjunction with diagnostic imaging, history, and physical examination. But in contrast to EGTM and ASCO, the National Academy of Clinical Biochemistry (NACB, 2008) Panel recommends against routine CA 15-3 or CEA testing in the surveillance of asymptomatic patients following diagnosis of operable breast cancer. And the National Comprehensive Cancer Network (NCCN, 2012) indicated that there is no evidence to support the use of "tumor markers" for breast cancer. 
Our results conclude elevated preoperative values of CA 15-3 was independent prognostic factors for OS, and overall survival were significantly shorter among patients with elevated serum CA15-3 or TPS. Which are supported by several studies with large patient groups and long follow-up times. B.-W. Park (2008) et al. found elevated preoperative CA 15-3 level is directly related to tumor burden and is independent prognostic factors for breast cancer, and Roland Einarsson (1995) found that pretreatment levels of TPS in patients with advanced breast cancer are related with prognosis. But there still others reported conflicting data: Sven Bornhak (2007) et al. proved that regular imaging and laboratory tests have no relevant effect on overall survival of patients of early breast cancer. And in another study of 400 patients, Stephane Zervoudis (2007) et al. concluded the value of increased tumor markers should be interpreted cautiously because it doesn't always imply disease progression, they may also increase in many benign conditions. Moreover, our finding showed that preoperative CEA concentration may not strongly suggest a better or worse prognosis. And Lumachi Franco (2010) et al. reported the baseline level of CEA is not very useful in prediction in elderly patients with breast cancer. But François-Clément Bidard (2012) et al. indicated CEA was independently associated with PFS (progression-free survival), which had globally similar performances.

The reason for these differences is not clearly understood currently. We inferred that the difference in study populations, detecting methods, related standards, result interpretations, control key points, prevention measures, and so on might all confound the results. While retrospective nature may be the main limitation of our study. Because any bias related to clinical decisions, which is not formulated beforehand, may causes tremendous influence.

Overall, despite some controversies, these serum tumor markers as easy, quick, cheap, noninvasive, but rather imprecise and sometimes misleading tools showed considerable prognostic value in untreated breast cancer patients. Since our analysis focused only on early risk profiles, these serum tumor markers may facilitate the postoperative follow-up for an early diagnosis of recurrence and for monitoring response to treatment. We have reason to believe when tumor marker determinations are applied in a proper way in the appropriate situation, the results can assist the oncologist enormously.

Furthermore, what should be mentioned slightly is the case number of triple-negative breast cancer, which is one type of immunohistological subtypes, is too small to draw any conclusion now. This is subjected to further expand the number of cases in the future.

\section{Acknowledgements}

We are grateful for the unselfish help of innumerable anonymous patients, physicians, nurses, and data managers who had contributed to this study.

\section{References}

Ahn, S. H., Yoo, K. Y., \& The Korean Breast Cancer Society. (2006). Chronological changes of clinical characteristics in 31115 new breast cancer patients among Koreans during 1996-2004. Breast Cancer Research and Treatment, 99(2), 209-214. http://dx.doi.org/10.1007/s10549-006-9188-x

Bidard, F.-C., Hajage, D., Bachelot, T., Delaloge, S., Brain, E., Campone, M., ... Pierga, J.-Y. (2012). Assessment of circulating tumor cells and serum markers for progression-free survival prediction in metastatic breast cancer: a prospective observational study. Breast Cancer Research, 14(1), R29. http://breast-cancer-research. com/content/14/1/R29

Bornhak, S., Heidemann, E., Herschlein, H.-J., Simon, W., Merkle, E., Widmaier, G., ... Brinkmann, F. (2007). Symptom-oriented follow-up of early breast cancer is not inferior to conventional control. Results of a prospective multicentre study. Onkologie, 30, 443-449. http://dx.doi.org/10.1159/000105257

Carison, R. W., Allred, D. C., Anderson, B. O., Burstein, H. J., Edge, S. B., Farrar, W. B., ... Zellars, R. (2012). NCCN Clinical Practice Guidelines in Oncology - Breast Cancer V1.2012. National Comprehensive Cancer Network, 2012, January. http://www.nccn.org/professionals/physician_gls/f_guidelines.asp\#breast

Einarsson, R. (1995). TPS - A cytokeratin marker for therapy control in breast cancer. Scandinavian Journal of Clinical \& Laboratory Investigation, 55, 113-115. http://dx.doi.org/10.3109/00365519509090574

Elmore, J. G., Barton, M. B., Moceri, V. M., Polk, S., Arena, P. J., \& Fletcher, S. W. (1998). Ten-year risk of false positive screening mammograms and clinical breast examinations. New England Journal of Medicine, 338(16), 1089-1096. http://dx.doi.org/10.1056/NEJM199804163381601

Harris, L., Fritsche, H., Mennel, R., Norton, L., Ravdin, P., Taube, S., .. Bast Jr, R. C. (2007). American Society of Clinical Oncology 2007 update of recommendations for the use of tumor markers in breast cancer. 
Journal of clinical oncology, 25(33), 5287-5312. http://dx.doi.org/10.1200/JCO.2007.14.2364

Hwa, H. L., Kuo, W. H., Chang, L. Y., Wang, M. Y., Tung, T. H., Chang, K. J., \& Hsieh F.-J. (2008). Prediction of breast cancer and lymph node metastatic status with tumour markers using logistic regression models. Journal of Evaluation in Clinical Practice, 14(2), 275-280. http://dx.doi.org/10.1111/j.1365-2753.2007.00 849.x

Lumachi, F., Basso, S. M., Bonamini, M., Marzano, B., Milan, E., Waclaw, B. U., \& Chiara G. B. (2010). Relationship between preoperative serum markers CA 15-3 and CEA and relapse of the disease in elderly ( $>65$ years) women with breast cancer. AnticancerResearch, 30(6), 2331-2334. http://ar.iiarjournals.org/cont ent/30/6/2331.short

Molina, R., Barak, V., Dalen, A., Duffy, M. J., Einarsson, R., Gion, M., ... Stieber, P. (2005). Tumor Markers in Breast Cancer - European Group on Tumor Markers Recommendations. Tumor Biology, 26, 281-293. http://dx.doi.org/10.1159/000089260

Park, B.-W., Oh, J.-W., Kim, J.-H., Park, S. H., Kim, K.-S., Kim, J. H., \& Lee, K. S. (2008). Preoperative CA 15-3 and CEA serum levels as predictor for breast cancer outcomes. Annals of Oncology, 19, 675-681. http://dx. doi.org/10.1093/annonc/mdm538

Rydlander, L., Ziegler, E., Bergman, T., Schöberl, E., Steiner, G., Bergman, A. C., ... Jörnvall, H. (1996). Molecular characterization of a tissue-polypeptide-specific-antigen epitope and its relationship to human cytokeratin 18. European Journal of Biochemistry, 241(2), 309-314. http://dx.doi.org/10.1111/j.1432-1033 .1996.00309.x

Siegel, R., Naishadham, D., \& Jemal, A. (2012). Cancer statistics, 2012. CA: A Cancer Journal for Clinicians, 62(1), 10-29. http://dx.doi.org/10.3322/caac.20138

Sturgeon, C. M., Duffy, M. J., Stenman, U. H., Lilja, H., Brünner, N., Chan, D. W., ... Diamandis, E. P. (2008). National Academy of Clinical Biochemistry Guidelines for the use of tumor markers in testicular, prostate, colorectal, breast, and ovarian cancers. Clinical Chemistry, 54(12), e11-79. http://dx.doi.org/10.1373/clinch em.2008. 105601

Uehara, M., Kinoshita, T., Hojo, T., Akashi-Tanaka, S., lwamoto, E., \& Fukutomi, T. (2008). Long-term prognostic study of carcinoembryonic antigen (CEA) and carbohydrate antigen 15-3 (CA15-3) in breast cancer. International Journal of Clinical Oncology, 13(5), 447-451. http://dx.doi.org/10.1007/s10147-0080773-3

Yerushalmi, R., Tyldesley, S., Kennecke, H., Speers, C., Woods, R., Knight, B., \& Gelmon, K. A. (2011). Tumor markers in metastatic breast cancer subtypes: frequency of elevation and correlation with outcome. Annals of Oncology, 23(2), 338-345. http://dx.doi.org/10.1093/annonc/mdr154

Zervoudis, S., Peitsidis, P., Iatrakis, G., Panourgias, E., Koureas, A., Navrozoglou, I., \& Dubois, J. B. (2007). Increased levels of tumor markers in the follow-up of 400 patients with breast cancer without recurrence or metastasis: interpretation of false-positive results. Journal of the Balkan Union of Oncology, 12(4), 487-492. http://www.bu-on.org/jbuon/article.php?id=453 - 5.5kb 